Review

\title{
Interleukin-10 Family and Tuberculosis: An Old Story Renewed
}

\author{
Abualgasim Elgaili Abdalla1,2, Nzungize Lambert' ${ }^{1}$, Xiangke Duan $^{1}$, Jianping Xie ${ }^{1 凶}$ \\ 1. Institute of Modern Biopharmaceuticals, State Key Laboratory Breeding Base of Eco-Environment and Bio-Resource of the Three Gorges Area, Key \\ Laboratory of Eco-environments in Three Gorges Reservoir Region, Ministry of Education, School of Life Sciences, Southwest University, Beibei, Chongqing \\ 400715, China. \\ 2. Department of Clinical Microbiology, College of Medical Laboratory Sciences, Omdurman Islamic University, Omdurman, Khartoum, Sudan. \\ $\triangle$ Corresponding author: Jianping Xie E-mail: georgex@swu.edu.cn Tel\&Fax: 862368367108.
}

(C) Ivyspring International Publisher. Reproduction is permitted for personal, noncommercial use, provided that the article is in whole, unmodified, and properly cited. See http://ivyspring.com/terms for terms and conditions.

Received: 2015.09.17; Accepted: 2016.01.15; Published: 2016.04.27

\begin{abstract}
The interleukin-10 (IL-10) family of cytokines consists of six immune mediators, namely IL-10, IL-19, IL-20, IL-22, IL-24 and IL-26. IL-10, IL-22, IL-24 and IL-26 are critical for the regulation of host defense against Mycobacterium tuberculosis infections. Specifically, IL-10 and IL-26 can suppress the antimycobacterial immunity and promote the survival of pathogen, while IL-22 and IL-24 can generate protective responses and inhibit the intracellular growth of pathogen. Knowledge about the new players in tuberculosis immunology, namely IL-10 family, can inform novel immunity-based countermeasures and host directed therapies against tuberculosis.
\end{abstract}

Key words: IL-10; cytokine; Mycobacterium tuberculosis; Immunity.

\section{Introduction}

Tuberculosis(TB), caused by Mycobacterium tuberculosis $(M t b)$, remains a leading cause of morbidity and mortality globally [1]. The chronicity of tuberculosis mirrors the complex interaction between the pathogen and the host [2]. The HIV coinfection and emergency of multidrug and extensive drug resistant $M t b$ further exacerbated the predicament of tuberculosis control [3]. Mycobacterium bovis Bacillus Calmette-Guerin (BCG), the only vaccine available for $\mathrm{TB}$, was plagued by its limited and sometime unstable efficacy [4].

Cytokines are critical for immunity against foreign invaders [5]. The interleukin (IL)-10 family of cytokines includes six immune mediators (IL-10, IL-19, IL-20, IL-22, IL-24 and IL-26) with similar protein motif, receptor complex and conserved signaling cascades [6, 7]. Their biological effect is diverse and widely ranges from anti-inflammatory to pro-inflammatory [6]. Two subfamilies exist based on the biological effect, immune regulatory cytokines including IL-10 only, and IL-20 subfamily consisting of the remaining five members (IL-19, IL-20, IL-22,
IL-24 and IL-26) [8]. The genes encoding IL-10, IL-19, IL-20 and IL-24 are located on the chromosome 1q32 [6]. Whereas, IL-22 and IL-26 encoding genes cluster with IFN-gamma gene on the chromosome 12q15 [6]. IL-10 signals via IL-10RA/IL-10RB [9] and results in the phosphorylation of Stat3, a critical transcription factor for the immunosuppressive effect of IL-10 [10]. IL-19, IL-20 and IL-24 induced their signaling through the IL-20RA/IL-20RB receptor complex. However, IL-20 and IL-24 preferentially engage with the IL22RA1/IL-20RB receptor complex. IL-22 and IL-26 function via receptor complex consisting of IL-22RA1/IL-10RB or IL-20RA/IL-10RB [11]. Interestingly, IL-20 subfamily can also activate Stat3, but the outcome is different from the activation of IL-10 [11]. The molecular mechanisms underlying the diverse effect of Stat3 signaling remain poorly understood.

The role of IL-10 family of cytokines in tuberculosis has been addressed recently [12-16]. Here we discussed the roles of IL-10, IL-22, IL-24 and IL-26 in the context of tuberculosis. 


\section{Cellular source and mycobacterial regulation of IL-10 family of cytokine.}

IL-10 can be produced by hematopoietic and non-hematopoietic cells in response to Mycobacterium tuberculosis Complex infection (Figure 1). BCG can activate the pattern recognition receptor (PRR) on the surface of alveolar epithelial cells. The engagement of TLR2 and TLR4 on surface of epithelial cells by BCG can activate the glycogen synthase kinase-3 (GSK3) by PI3K-Akt and promote the production of IL-10 and IL-22 [17]. However, conflicting evidence is that the suppression of GSK3 by chemical inhibitor can significantly boost the BCG induction of IL-10 derived from macrophages [18].

In neutrophils, coactivation of TLR2-MyD88and C-type lectin receptor (CLR)- spleen tyrosine kinase (Syk)-dependent pathways led to the phosphorylation of p38 MAP and Akt kinases and subsequently, the upregulation of IL-10 [19].

The interaction between the BCG-infected dendritic cells (DCs) with neutrophil through CD11b can activate MyD88 and Syk, to produce large quantities of IL-10 [20].

$M t b$ can upregulate the IL-10 production via TLR2- ERK pathway [21]. Activation of ERK by $M t b$ Rv1265 was critical for induced IL-10 expression in macrophages [22]. Binding of the $M t b$ heat shock protein 60 to the TLR2 can activate macrophage p38 MAPK to increase the IL-10 production [23]. Similarly, the engagement of Mtb PPE32 with TLR2 can upregulate the IL-10 production via co-activation of
NF-kB and MAPK [24]. Taken together, TLR2 and its downstream signaling MAPK pathway are essential for the induction of IL-10 expression in myeloid cells in response to the $M$. tuberculosis infection [25].

TLR3 regulates IL-10 expression in response to Mycobacterial-RNA through the PI3K/AKT signaling pathway [26]. BCG-stimulated IL-10 expression in human macrophages by activation of dsRNA-activated serine/threonine protein kinase (PKR), which in turn activates MAPK and NF-K $\beta$ [27].

$M t b$ promoted chemokine receptor 5 (CCR5) expression in macrophages, which in turn increased IL-10 production [28].

It was found that IFN-a can induce IL-10 production by macrophages through the phosphorylation of Stat1 [29]. IFN- $\alpha / \beta$ can induce IL-10 production by $M t b$-infected macrophages [30]. This was supported by a murine study demonstrating that suppression of IFN- $\alpha / \beta$ by TPL-2-ERK1/2 signaling led to inhibition of IL-10 production [31].

$M t b$ infected antigen-presenting cells (APCs) can activate DAP12 (DNAX-activating protein of $12 \mathrm{kDa}$ ) required for IRAK-M (interleukin-1 receptorassociated kinase $\mathrm{M}$ ) expression, which in turn induces IL-10 production [32]. BCG can enhance the production of IL-10 by binding to the DC-SIGN in DCs [33]. Similarly, Mannose-capped lipoarabinomannan (Man-LAM) can induce IL-10 production through targeting the Dectin-2 in APCs [34]. BCG induced high levels of IL-10 production by cord blood DCs via upregulation of the nuclear transcription factor Rel-B [35].
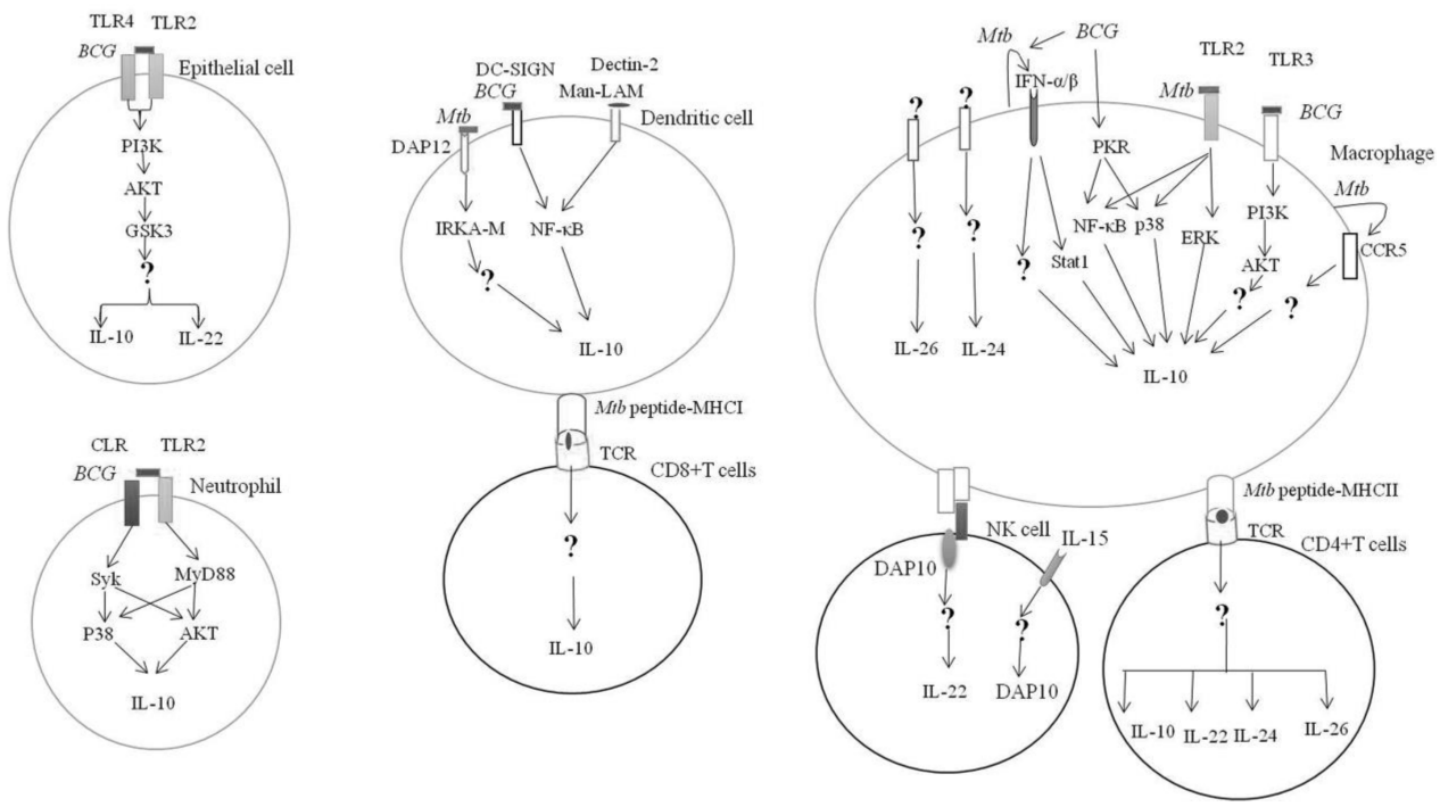

Figure 1. Mycobacterial regulation of IL-10 family expression. Mycobacterium tuberculosis or M.bovis BCG can engage with PRR on the hematopoietic and non-hematopietic cells and result in the activation of downstream effectors and IL-10 expression. Receptors and signaling involved in the expression of IL-20 subfamily in response to Mtb infection is currently unknown. $\rightarrow$ Direct activation; $\lrcorner=$ autocrine; ? = unknown 
$M t b$ can induce IL-10 production by CD8+ T cells $[36,37]$. CD4+ T cells produced high levels of IL-10 in response to purified protein derivative (PPD) [38].

IL-22 is produced by cells of the lymphoid lineages in response to Mycobacteria, which includes CD4+ cells (Th17 and Th22) [39, 40] and natural killer (NK) cells [41] (Figure 1).

Stimulation of NK cells with $M t b$ in the presence of IL-15 led to DAP10 expression and subsequently, IL-10 production [42]. Mtb PstS1 upregulated IL-22 secretion from memory $\mathrm{CD} 4+\mathrm{T}$ was mediated via DCs [43].

However, regulatory B cells can suppress IL-22 production [44]. The production of IL-22 showed significant increase in the absence of IFN- $\gamma$ [45].

IL-24 is mainly produced by monocytes and activated T cells [46, 47](Figure 1).

IL-26 is produced by myeloid and lymphoid lineages of white blood cells in response to $M t b$ infection [13](Figure 1). IL-26 can be released by Th17 [48, 49], Th1 cells [48] and NK cells [50]. However, how $M t b$ or its antigens regulate the expression of IL-22, IL-24 and IL-26 remains to be elucidated.

\section{Role of IL-10 family in Mycobacterial immune evasion.}

IL-10 is a potent immunoregulatory cytokine indispensable for the host immunity against mycobacteria infection (Figure 2). Consistently, IL-10 transgenic mice fail to control $M t b$ infection and develop severe lung pathology compared with nontransgenic littermates [51]. However, IL-10-knockout mice were more resistant to $M t b$ infection and can limit bacterial growth within lung and spleen in comparison with the wild type [52]. Blocking of murine IL-10 signaling during $M t b$ infection was beneficial for the control of the growth of bacteria and enhanced the survival of mice [53]. It was shown that IL-10 -/- mice exhibited stronger Th1 responses during $M t b$ infection with concomitant massive pulmonary inflammation [54]. However, conflicting data is reported on the immunomodulatory effect of IL-10 during Mycobacterial infection. IL-10 transgenic mice show normal Th1 response to $M t b$ infection, but IL-10 can reduce the production of antimicrobials reactive nitrogen intermediates (NO) by macrophages via increased arginase-1 (arg1) expression. This led to the progression of the disease and earlier death of mice [55]. Furthermore, the expression of arg1 in hypoxic granuloma regions led to decreased $\mathrm{T}$ cells expansion [56].

The absence of IL-10 enhanced the production of IL-17, and resulted in excessive lung inflammatory response [12]. Similarly, blockade of IL-10 during
BCG vaccination can increase the protection against $M t b$ challenge in both susceptible and comparatively resistant strains of mice due to enhanced Th17 cell responses [57]. IL-10 can repress 17A production through direct interacting with IL-10R $\alpha$ in Th17 cell [20]. IL-17A deficient mice failed to develop mature granuloma and protective responses against virulent $M t b$ infection [58]. However, IL-10-deficient mice infected with $M t b$ can generate mature fibrotic pulmonary granulomas and improve the control of bacterial growth in the lungs [16]. Furthermore, IL-10 suppresses TNF- $\alpha$ production $[30,59,60]$, TNF- $\alpha$ is essential for granulomas formation and blocking of TNF- $\alpha$ signaling leads to the disintegration of granuloma and dissemination of $M t b$ [61]. Moreover, IL-10 can suppress the formation of multi-nucleated giant cells (MGC) from monocytes of TB patients in response to PPD [62], the histologic hallmark of granuloma which can limit $M t b$ infection. Taken together, the data suggest that IL-10 promotes the persistence and survival of pathogens. Therefore, the modulation of IL-10 levels might facilitate the TB elimination.

Peripheral blood mononuclear cells (PBMCs) obtained from multi-drug resistant TB patients stimulated by ESAT6 displayed a lower frequency of IFN- $\gamma$-producing $\mathrm{T}$ cells and a higher frequency of regulatory $\mathrm{T}$ cells (Treg) correlated with increased IL-10 secretion, when compared with cells from non-tuberculous Mycobacteria patients. Blockage of IL-10 or IL-10Ra with antibody can enhance Th1 responses and decreases the Treg cells [63].

Macrophages apoptosis is important for the cross-priming of T cells by DCs. DCs can uptake the apoptotic vesicle from the infected apoptotic macrophages and subsequently prime the naïve $\mathrm{T}$ cells [64]. IL-10 can suppress $M t b$-induced macrophages apoptosis by reducing TFN- $\alpha$ and upregulating of $\mathrm{Bcl} 3$, an inhibitor of NF- $\mathrm{KB}$ activity [60]. Similarly, inhibition of macrophages apoptosis by virulent $M$. bovis was shown to be correlated with increased IL-10 and Bcl2 production and decreased TNF-a production [65]. Taken together, Mycobacteria can subvert the apoptosis of macrophages for better survival and persistence.

IL-10 can inhibit the production of IL-12 in $M t b$-infected macrophages [30]. IL-10 can interfere with antigen presentation by inducing MARCH1 member of ubiquitin ligase family that interacts and degrades class II major histocompatibility complex (MHC-II) molecules [66]. It has been shown that IL-10 pretreated monocyte-derived macrophages (MDMs) failed to traffic the Mtb peptide-MHC-II complexes to the plasma membrane [67]. IL-10 also inhibits MHC-II expression in $M t b$-infected macrophages via 
activation of Stat3 [28]. Suppression of IL-12 and MHC-II by IL-10 in Mtb-infected macrophages led to compromised the Th1 responses [21]. Consistently, blocking of IL-10 signaling in $M t b$-infected macrophages can enhance IL-12 secretion and augment the IFN- $\gamma$ production by CD4+ T cells [68].

IL-10 also can enhance Mtb survival and growth in macrophages by suppressing phagosomal maturation partially dependent on Stat 3 activation [69]. Taken together, IL-10 enhances mycobacterial intracellular survival and growth by suppressing innate and adaptive immune response.

The role of IL-26 in host defense against $M t b$ has been explored very recently (Figure 2). The possible role of IL-26 in $M t b$ infection has been demonstrated by ex vivo study [13]. Levels of IL-26 are lower in the $M t b$-infected monocytes supernatants from TB patients than from healthy control [13]. The addition of recombinant IL-26 to whole blood infected with $M t b$ led to enhanced bacterial survival and growth [13]. However, how Mtb downregulates IL-26 and its role in antimycobacterial response remain to be explored.

IL-26 has quite unexpected cationic antimicrobials peptides properties that can lyse extracellular bacteria by forming membrane-pore [70]. Also, it can bridge the released bacterial DNA and TLR9, thereby triggering the production of type I IFN by plasmacytoid DCs [70]. IFN- $\alpha / \beta$ are potent inhibitors of the IFN- $\gamma$ responsiveness and the production of type I cytokines that are required for protection against $M t b$ infection [48].

IL-26 produced by alveolar macrophages can recruit the neutrophils via induction of chemotaxis factors such IL-8 and leukotaxin n-formyl-methionyl-leucyl-phenylalanine (fMLP) [71]. IL-26 also can inhibit random migration (chemokinesis) of neutrophils [71]. Depletion of the neutrophil at the onset of $\mathrm{TB}$ infection results in significant increase the numbers of IFN- $\gamma$-producing CD4 T-cells, correlated with reduced bacterial load and lung pathology [72]. In-depth understanding of IL-26 biology is crucial to the development of immune intervention against tuberculosis.

\section{Role of IL-10 family in immune protective against Mycobacteria infection.}

It was predicted that the production of low levels of IL-10 by activated macrophages is required to an efficient antimicrobial response, control of bacterial growth and to prevent lung damage [73]. Therefore, modulation of IL-10 levels during TB therapy might shorten the duration of the treatment and accelerate the bacterial clearance.

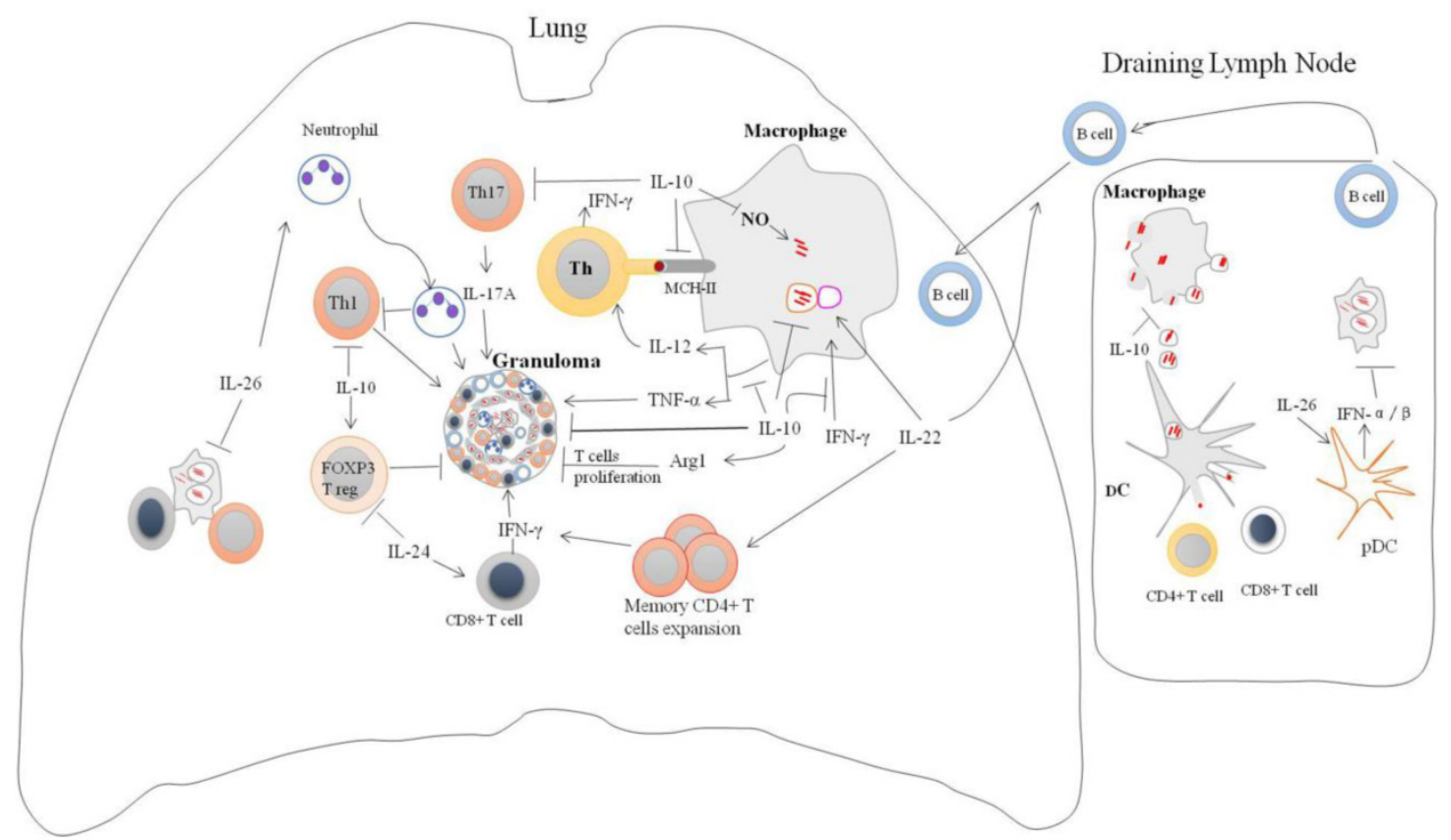

Figure 2. Regulation of immune responses by IL-10 family cytokines during Mycobacteria infection: IL-10 family of cytokines induced by mycobacteria infection modulates immune responses. IL-10 can enhance intracellular survival and growth of bacilli by inhibition nitric oxide production, phagosomal maturation and IFN- $\gamma$-mediating killing. In addition, IL-10 can inhibit CD4+ T cell responses by suppression of IL-12 production and trafficking of Mtb peptide-MHC-II complexes to the plasma membrane. It also suppresses $\mathrm{T}$ cells cross-priming by antigen-presenting cells via blocking macrophages apoptosis. IL-10 can modulate granuloma formation via inhibition of TNF- $\alpha$, Th17 cells and T cells expansion. Il-26 enhances intracellular survival of Mtb by unknown mechanism. IL-26 can recruit neutrophils to site of battle that can cause tissue damage and suppression Th1 response. IL-26 also promotes binding of released bacterial DNA to the TLR9 in surface of plasmacytoid DC resulting in production type I IFN. IL-22 can inhibit intracellular growth of bacilli via enhancing phagolysosomal fusion. IL-22 is an important activator of adaptive immune responses through promoting the expansion of memory CD4+ cells and IFN-Y production and recruitment of B cells to site of infection. IL-24 induces IFN-Y production by CD8+ cells and suppresses FOXP3 T reg cells. 
IL-22 is crucial for tissues modulation in responses to inflammation. It induces proliferative and anti-apoptotic pathways, as well as anti-microbial substances, that can enhance tissue repair and prevent its damage [11].

IL-22 has been shown to be significantly upregulated in the blood from juvenile rhesus monkeys with severe TB disease [74]. IL-22-producing $\mathrm{T}$ cells were found most frequent localized in lungs than in blood and lymphoid tissue, and involved in granuloma formation in macaque TB model [15]. Interestingly, IL-22 was found to be most significantly upregulated in PBMCs from vaccinated-protected cattle, when compared with vaccinated-unprotected and unvaccinated control, suggested that IL-22 a potential biomarker for vaccine success [75]. IL-22-deficient mice display normal response to $M t b$ infection [76]. However, IL-22 may trigger the recruitment of $\mathrm{B}$ cells to the lungs by upregulating CXCL13 expression [77]. B cells play crucial roles in host defense by reducing inflammation and containment of $M t b$ [78]. IL-22-knockout mice challenged with $M t b$ exhibited smaller B cell follicles in comparison with the counterpart [77].

Administration of IL-22 into the mice at the time of BCG vaccination led to reduced bacterial burden and enhanced CD4+ $\mathrm{T}$ cell responses after $\mathrm{M} t b$ challenge [41].

A polymorphism (rs2227473) in the IL-22 promoter was shown to be associated with TB susceptibility in Chinese populations [79]. IL-22 producing CD4+ $\mathrm{T}$ cells (Th22 ) frequencies were lowered in PBMCs from TB pleurisy (PTB) patients in comparison with healthy, whereas, IL-22 was detected in high concentration in bronchoalveolar lavage (BAL) fluid obtained from TB patients [80]. Likewise, the Th22 cells are elevated in tuberculous pleural fluid [40, 81], suggested that IL-22 does involve in immunity at local site of $M t b$ infection. In HIV patients with TB disease, IL-22 was differentially induced in PBMCs collected from TB with reconstitution inflammatory syndrome (TB-IRIS) after in vitro stimulation with $M t b$, and higher concentration of IL-22 proteins was detected in their serum, when compared with non-IRIS patients [82]. Taken together, these data suggest that IL-22 might play a decisive role in tuberculosis immunity.

Modern Beijing strains can stimulate PBMCs from healthy individuals to produce lower level of IL-22 than the ancient Beijing and Euro-American strains [83]. Manipulation of IL-22 level by $M t b$ modern Beijing strains might benefit its intracellular survival.

IL-22 plays indispensable role in innate and adaptive immune defense against $M t b$ infection
(Figure 2), production of IL-22 by NK cells can inhibit intracellular growth of $M t b$ through enhancing phagolysosomal fusion [42]. Interesting, IL-22 can be found in the membrane of mature CD4+ $\mathrm{T}$ effector cells and the direct interaction of these cells with Mtb-infected macrophages led to suppress intracellular bacterial growth [84]. However, the molecular mechanisms by which IL-22 inhibits intracellular growth of $M t b$ are to be determined. Recently, it was found that IL-22 induced calgranulin A expression that can enhance phagolysosomal fusion by increasing Rab7 expression and downregulatiing Rab14 expression [85].

IL-24, also known as melanoma differentiation associated gene 7 product(mda-7), has potent antitumor activity through selectively induced tumor death without detriment effect in normal cells [86]. Levels of IL-24 are significantly lower in the serum of TB patients in comparison with healthy volunteers [14]. IL-24 expression was decreased in PBMCs infected with Mtb, and addition of recombinant IL-24 can induce IFN- $\gamma$ production by CD8+ T cells [14].

IL-24 was significantly upregulated in PBMCs obtained from BCG-Japan strain-vaccinated children stimulated with culture filtrate proteins (CFP) of $M t b$ compared with children vaccinated with BCG-Brazil or BCG-Denmark strain [87]. PBMCs obtained from latent TB patients (LTB) produces more IL-24 than from active TB patients [88]. Furthermore, exogenous IL-24 can increase IFN- $\gamma$ in PBMCs obtained from TB patients, however, neutralization of IL-24 can reduce IFN- $\gamma$ expression in PBMCs collected from LTB patients [46]. IL-24 increased IFN- $\gamma$ expression through increasing expressions of IL-12, 1L-23 and IL-27, and by decreasing FOXP3 expression [46] (Figure 2). Taken together, $M t b$ suppresses IL-24 expression might enhance its persistence host tissue. However, underlying molecular mechanisms remain to be explored.

\section{Conclusions}

In conclusion, IL-10 family of cytokines has diverse roles during Mycobacteria infection ranging from immune evasion to immune protective. The absence of IL-10 can enhance immune response and early clearance of $M t b$. IL-10 can enhance the intracellular survival of bacilli via several means, namely 1) inhibiting phagosomal maturation, 2) reducing nitric oxide production and 3) blocking IFN$\gamma$ signaling in macrophage. In addition, IL-10 can antagonize the development of Th1 responses through suppressing antigen presentation and IL-12 production. Moreover, it can inhibit macrophages apoptosis that is require for efficient development of $\mathrm{T}$ cells responses. However, low level IL-10 can trigger 
antimicrobial response and prevent tissue damage. Therefore, decreasing the IL-10 level during TB therapy might benefit for the rapid clearance of bacteria.

IL-26 is a novel player with scarce information in TB immune responses; it can limit $M t b$ clearance during in vitro infection of PBMCs by mechanisms currently unknown. Understanding how IL-26 enhances intracellular growth of bacilli is critical to develop better countermeasures against this notorious pathogen.

IL-22 plays a decisive role in innate and adaptive immune responses against $M t b$ infection by promoting phagolysosomal fusion, expansion and IFN- $\gamma$ production by memory CD4+ cells and recruitment of B cells.

IL-24 induces IFN- $\gamma$ production by CD8+ T cells. IL-22 and IL-24, crucial for the coordinated cell-mediated immunity, might represent good candidates for better TB therapy.

\section{Acknowledgement}

Abualgasim E Abdalla is supported by China government scholarship (No. 2013736003) for the PhD degree supervised by Professor Jianping Xie.

This work was supported by the National Natural Science Foundation (grant numbers 81371851, 8151120001，81071316， 81271882,81301394), New Century Excellent Talents in Universities (grant number NCET-11-0703), National Megaprojects for Key Infectious Diseases (grant number 2008ZX10003-006), Excellent PhD thesis fellowship of Southwest University (grant numbers kb2010017, ky2011003), the Fundamental Research Funds for the Central Universities (Nos. XDJK2011D006, XDJK2012D011，XDJK2012D007, XDJK2013D003 and XDJK2014D040), Natural Science Foundation Project of CQ CSTC (grant number CSTC 2010BB5002), The Chongqing municipal committee of Education for postgraduate excellence program (No.YJG123104), the undergraduate teaching reform program (No. 2011JY052).

\section{Competing Interests}

The authors have declared that no competing interest exists.

\section{References}

1. Walzl G, Haks MC, Joosten SA, Kleynhans L, Ronacher K, Ottenhoff TH. Clinical Immunology and Multiplex Biomarkers of Human Tuberculosis. Cold Spring Harb Perspect Med. 2014.

2. Abdalla AE, Li Q, Xie L, Xie J. Biology of IL-27 and its Role in the Host Immunity against. Int J Biol Sci. 2015; 11: 168-75.

3. Bhembe NL, Nwodo UU, Govender S, Hayes C, Ndip RN, Okoh AI, et al. Molecular detection and characterization of resistant genes in Mycobacterium tuberculosis complex from DNA isolated from tuberculosis patients in the Eastern Cape province South Africa. BMC Infect Dis. 2014; 14: 479.
4. Evans TG, Brennan MJ, Barker L, Thole J. Preventive vaccines for tuberculosis. Vaccine. 2013; 31 Suppl 2: B223-6.

5. Ouyang W, Rutz S, Crellin NK, Valdez PA, Hymowitz SG. Regulation and functions of the IL-10 family of cytokines in inflammation and disease. Annu Rev Immunol. 2011; 29: 71-109.

6. Sabat R. IL-10 family of cytokines. Cytokine Growth Factor Rev. 2010; 21: 315-24.

7. Zdanov A. Structural analysis of cytokines comprising the IL-10 family. Cytokine Growth Factor Rev. 2010; 21: 325-30.

8. Hofmann SR, Rosen-Wolff A, Tsokos GC, Hedrich CM. Biological properties and regulation of IL-10 related cytokines and their contribution to autoimmune disease and tissue injury. Clin Immunol. 2012; 143: 116-27.

9. Acuner-Ozbabacan ES, Engin BH, Guven-Maiorov E, Kuzu G, Muratcioglu S, Baspinar A, et al. The structural network of Interleukin-10 and its implications in inflammation and cancer. Bmc Genomics. 2014; 15.

10. Hutchins AP, Diez D, Miranda-Saavedra D. The IL-10/STAT3-mediated anti-inflammatory response: recent developments and future challenges. Brief Funct Genomics. 2013; 12: 489-98.

11. Rutz S, Wang XT, Ouyang WJ. The IL-20 subfamily of cytokines - from host defence to tissue homeostasis. Nat Rev Immunol. 2014; 14: 783-95.

12. Redford PS, Boonstra A, Read S, Pitt J, Graham C, Stavropoulos E, et al. Enhanced protection to Mycobacterium tuberculosis infection in IL-10-deficient mice is accompanied by early and enhanced Th1 responses in the lung. Eur J Immunol. 2010; 40: 2200-10.

13. Guerra-Laso JM, Raposo-Garcia S, Garcia-Garcia S, Diez-Tascon C, Rivero-Lezcano OM. Microarray analysis of Mycobacterium tuberculosis-infected monocytes reveals IL26 as a new candidate gene for tuberculosis susceptibility. Immunology. 2015; 144: 291-301.

14. Ma Y, Chen HD, Wang Y, Wang Q, Li Y, Zhao Y, et al. Interleukin 24 as a novel potential cytokine immunotherapy for the treatment of Mycobacterium tuberculosis infection. Microbes Infect. 2011; 13: 1099-110.

15. Yao S, Huang D, Chen CY, Halliday L, Zeng G, Wang RC, et al. Differentiation, distribution and gammadelta $\mathrm{T}$ cell-driven regulation of IL-22-producing T cells in tuberculosis. PLoS Pathog. 2010; 6: e1000789.

16. Cyktor JC, Carruthers B, Kominsky RA, Beamer GL, Stromberg P, Turner J. IL-10 inhibits mature fibrotic granuloma formation during Mycobacterium tuberculosis infection. J Immunol. 2013; 190: 2778-90.

17. Lutay N, Hakansson $\mathrm{G}$, Alaridah $\mathrm{N}$, Hallgren $\mathrm{O}$, Westergren-Thorsson $\mathrm{G}$, Godaly G. Mycobacteria bypass mucosal NF-kB signalling to induce an epithelial anti-inflammatory IL-22 and IL-10 response. PLoS One. 2014; 9: e86466.

18. Chan MM, Cheung BK, Li JC, Chan LL, Lau AS. A role for glycogen synthase kinase-3 in antagonizing mycobacterial immune evasion by negatively regulating IL-10 induction. J Leukoc Biol. 2009; 86: 283-91.

19. Zhang X, Majlessi L, Deriaud E, Leclerc C, Lo-Man R. Coactivation of Syk kinase and MyD88 adaptor protein pathways by bacteria promotes regulatory properties of neutrophils. Immunity. 2009; 31: 761-71.

20. Doz E, Lombard R, Carreras F, Buzoni-Gatel D, Winter N. Mycobacteria-infected dendritic cells attract neutrophils that produce IL-10 and specifically shut down Th17 CD4 T cells through their IL-10 receptor. J Immunol. 2013; 191: 3818-26.

21. Richardson ET, Shukla S, Sweet DR, Wearsch PA, Tsichlis PN, Boom WH, et al. Toll-like receptor 2-dependent extracellular signal-regulated kinase signaling in Mycobacterium tuberculosis-infected macrophages drives anti-inflammatory responses and inhibits Th1 polarization of responding $\mathrm{T}$ cells. Infect Immun. 2015; 83: 2242-54

22. Luo H, Zeng J, Huang Q, Liu M, Abdalla AE, Xie L, et al. Mycobacterium tuberculosis Rv1265 promotes mycobacterial intracellular survival and alters cytokine profile of the infected macrophage. J Biomol Struct Dyn. 2015: 1-15.

23. Parveen N, Varman R, Nair S, Das G, Ghosh S, Mukhopadhyay S. Endocytosis of Mycobacterium tuberculosis heat shock protein 60 is required to induce interleukin-10 production in macrophages. J Biol Chem. 2013; 288: 24956-71.

24. Deng W, Li W, Zeng J, Zhao Q, Li C, Zhao Y, et al. Mycobacterium tuberculosis PPE family protein Rv1808 manipulates cytokines profile via co-activation of MAPK and NF-kappaB signaling pathways. Cell Physiol Biochem. 2014; 33: 273-88.

25. Giacomini E, Remoli ME, Scandurra M, Gafa V, Pardini M, Fattorini L, et al. Expression of proinflammatory and regulatory cytokines via NF-kappaB and MAPK-dependent and IFN regulatory factor-3-independent mechanisms in human primary monocytes infected by Mycobacterium tuberculosis. Clin Dev Immunol. 2011; 2011: 841346.

26. Bai $W$, Liu $H$, Ji $Q$, Zhou $Y$, Liang L, Zheng $R$, et al. TLR3 regulates mycobacterial RNA-induced IL-10 production through the PI3K/AKT signaling pathway. Cell Signal. 2014; 26: 942-50.

27. Cheung BK, Lee DC, Li JC, Lau YL, Lau AS. A role for double-stranded RNA-activated protein kinase PKR in Mycobacterium-induced cytokine expression. J Immunol. 2005; 175: 7218-25.

28. Das S, Banerjee S, Majumder S, Chowdhury BP, Goswami A, Halder K, et al. Immune subversion by Mycobacterium tuberculosis through CCR 5 mediated signaling: involvement of IL-10. PLoS One. 2014; 9: e92477.

29. Ma J, Yang B, Yu S, Zhang Y, Zhang X, Lao S, et al. Tuberculosis antigen-induced expression of IFN-alpha in tuberculosis patients inhibits production of IL-1beta. FASEB J. 2014; 28: 3238-48.

30. McNab FW, Ewbank J, Howes A, Moreira-Teixeira L, Martirosyan A, Ghilardi $\mathrm{N}$, et al. Type I IFN induces IL-10 production in an IL-27-independent manner 
and blocks responsiveness to IFN-gamma for production of IL-12 and bacterial killing in Mycobacterium tuberculosis-infected macrophages. J Immunol. 2014; 193: 3600-12.

31. McNab FW, Ewbank J, Rajsbaum R, Stavropoulos E, Martirosyan A, Redford PS, et al. TPL-2-ERK1/2 signaling promotes host resistance against intracellular bacterial infection by negative regulation of type I IFN production. J Immunol. 2013; 191: 1732-43.

32. Jeyanathan M, McCormick S, Lai R, Afkhami S, Shaler CR, Horvath CN, et al. Pulmonary M. tuberculosis infection delays Th1 immunity via immunoadaptor DAP12-regulated IRAK-M and IL-10 expression in antigen-presenting cells. Mucosal Immunol. 2014; 7: 670-83.

33. Gagliardi MC, Teloni R, Giannoni F, Pardini M, Sargentini V, Brunori L, et al. Mycobacterium bovis Bacillus Calmette-Guerin infects DC-SIGN- dendritic cell and causes the inhibition of IL-12 and the enhancement of IL-10 production. J Leukoc Biol. 2005; 78: 106-13.

34. Yonekawa A, Saijo S, Hoshino Y, Miyake Y, Ishikawa E, Suzukawa M, et al. Dectin-2 is a direct receptor for mannose-capped lipoarabinomannan of mycobacteria. Immunity. 2014; 41: 402-13.

35. Liu E, Law HK, Lau YL. BCG promotes cord blood monocyte-derived dendritic cell maturation with nuclear Rel-B up-regulation and cytosolic I kappa B alpha and beta degradation. Pediatr Res. 2003; 54: 105-12.

36. Gerosa F, Nisii C, Righetti S, Micciolo R, Marchesini M, Cazzadori A, et al. $\mathrm{CD} 4(+) \mathrm{T}$ cell clones producing both interferon-gamma and interleukin-10 predominate in bronchoalveolar lavages of active pulmonary tuberculosis patients. Clin Immunol. 1999; 92: 224-34.

37. Cyktor JC, Carruthers B, Beamer GL, Turner J. Clonal expansions of CD8+ T cells with IL-10 secreting capacity occur during chronic Mycobacterium tuberculosis infection. PloS one. 2013; 8: e58612.

38. Boussiotis VA, Tsai EY, Yunis EJ, Thim S, Delgado JC, Dascher CC, et al. IL-10-producing $\mathrm{T}$ cells suppress immune responses in anergic tuberculosis patients. J Clin Invest. 2000; 105: 1317-25.

39. Li L, Qiao D, Fu X, Lao S, Zhang X, Wu C. Identification of M. tuberculosis-specific Th1 cells expressing CD69 generated in vivo in pleural fluid cells from patients with tuberculous pleurisy. PLoS One. 2011; 6: e23700.

40. Qiao D, Yang BY, Li L, Ma JJ, Zhang XL, Lao SH, et al. ESAT-6- and CFP-10-specific Th1, Th22 and Th17 cells in tuberculous pleurisy may contribute to the local immune response against Mycobacterium tuberculosis infection. Scand J Immunol. 2011; 73: 330-7.

41. Dhiman R, Periasamy S, Barnes PF, Jaiswal AG, Paidipally P, Barnes AB, et al. NK1.1+ cells and IL-22 regulate vaccine-induced protective immunity against challenge with Mycobacterium tuberculosis. J Immunol. 2012; 189: 897-905.

42. Dhiman R, Indramohan M, Barnes PF, Nayak RC, Paidipally P, Rao LV, et al. IL-22 produced by human NK cells inhibits growth of Mycobacterium tuberculosis by enhancing phagolysosomal fusion. J Immunol. 2009; 183: 6639-45.

43. Palma C, Schiavoni G, Abalsamo L, Mattei F, Piccaro G, Sanchez M, et al. Mycobacterium tuberculosis PstS1 amplifies IFN-gamma and induces IL-17/IL-22 responses by unrelated memory CD4+ T cells via dendritic cell activation. Eur J Immunol. 2013; 43: 2386-97.

44. Zhang M, Zeng G, Yang Q, Zhang J, Zhu X, Chen Q, et al. Anti-tuberculosis treatment enhances the production of IL-22 through reducing the frequencies of regulatory B cell. Tuberculosis (Edinb). 2014; 94: 238-44

45. Qiu Y, Huang Y, Chen J, Qiao D, Zeng G, Cai J. Depletion of IL-22 during culture enhanced antigen-driven IFN-gamma production by CD4(+)T cells from patients with active TB. Immunol Lett. 2013; 150: 48-53.

46. Wu B, Huang C, Kato-Maeda M, Hopewell PC, Daley CL, Krensky AM, et al. IL-24 modulates IFN-gamma expression in patients with tuberculosis. Immunology letters. 2008; 117: 57-62.

47. Yang BX, Duan YJ, Dong CY, Zhang F, Gao WF, Cui XY, et al. Novel functions for mda-7/IL-24 and IL-24 delE5: regulation of differentiation of acute myeloid leukemic cells. Mol Cancer Ther. 2011; 10: 615-25.

48. de Paus RA, van Wengen A, Schmidt I, Visser M, Verdegaal EM, van Dissel JT, et al. Inhibition of the type I immune responses of human monocytes by IFN-alpha and IFN-beta. Cytokine. 2013; 61: 645-55.

49. Wilson NJ, Boniface K, Chan JR, McKenzie BS, Blumenschein WM, Mattson JD, et al. Development, cytokine profile and function of human interleukin 17-producing helper T cells. Nat Immunol. 2007; 8: 950-7.

50. Cella M, Fuchs A, Vermi W, Facchetti F, Otero K, Lennerz JK, et al. A human natural killer cell subset provides an innate source of IL-22 for mucosal immunity. Nature. 2009; 457: 722-5

51. Turner J, Gonzalez-Juarrero M, Ellis DL, Basaraba RJ, Kipnis A, Orme IM, et al. In vivo IL-10 production reactivates chronic pulmonary tuberculosis in C57BL/ 6 mice. J Immunol. 2002; 169: 6343-51.

52. Turner J, Cyktor J, Carruthers B, Kominsky R, Stromberg P, Beamer G. Deficiency in interleukin 10 reverses the susceptibility phenotype of CBA/J mice during infection with Mycobacterium tuberculosis. J Immunol. 2012; 188.

53. Beamer GL, Flaherty DK, Assogba BD, Stromberg P, Gonzalez-Juarrero M, de Waal Malefyt $\mathrm{R}$, et al. Interleukin-10 promotes Mycobacterium tuberculosis disease progression in CBA/J mice. J Immunol. 2008; 181: 5545-50.

54. Higgins DM, Sanchez-Campillo J, Rosas-Taraco AG, Lee EJ, Orme IM, Gonzalez-Juarrero M. Lack of IL-10 alters inflammatory and immune responses during pulmonary Mycobacterium tuberculosis infection. Tuberculosis (Edinb). 2009; 89: 149-57.

55. Schreiber T, Ehlers S, Heitmann L, Rausch A, Mages J, Murray PJ, et al. Autocrine IL-10 induces hallmarks of alternative activation in macrophages and suppresses antituberculosis effector mechanisms without compromising T cell immunity. J Immunol. 2009; 183: 1301-12.

56. Duque-Correa MA, Kuhl AA, Rodriguez PC, Zedler U, Schommer-Leitner S, Rao M, et al. Macrophage arginase-1 controls bacterial growth and pathology in hypoxic tuberculosis granulomas. Proc Natl Acad Sci U S A. 2014; 111: E4024-32.

57. Pitt JM, Stavropoulos E, Redford PS, Beebe AM, Bancroft GJ, Young DB, et al. Blockade of IL-10 signaling during bacillus Calmette-Guerin vaccination enhances and sustains Th1, Th17, and innate lymphoid IFN-gamma and IL-17 responses and increases protection to Mycobacterium tuberculosis infection. J Immunol. 2012; 189: 4079-87.

58. Okamoto Yoshida Y, Umemura M, Yahagi A, O'Brien RL, Ikuta K, Kishihara $\mathrm{K}$, et al. Essential role of IL-17A in the formation of a mycobacterial infection-induced granuloma in the lung. J Immunol. 2010; 184: 4414-22.

59. Marshall BG, Coker RJ. Strategies for control of tuberculosis. Lancet. 1997; 349: 882-3.

60. Patel NR, Swan K, Li X, Tachado SD, Koziel H. Impaired M. tuberculosis-mediated apoptosis in alveolar macrophages from $\mathrm{HIV}+$ persons: potential role of IL-10 and BCL-3. J Leukoc Biol. 2009; 86: 53-60.

61. Yasui K. Immunity against Mycobacterium tuberculosis and the risk of biologic anti-TNF-alpha reagents. Pediatr Rheumatol Online J. 2014; 12: 45.

62. Shrivastava P, Bagchi T. IL-10 modulates in vitro multinucleate giant cell formation in human tuberculosis. PLoS One. 2013; 8: e77680.

63. Pinheiro RO, de Oliveira EB, Dos Santos G, Sperandio da Silva GM, de Andrade Silva BJ, Teles RM, et al. Different immunosuppressive mechanisms in multi-drug-resistant tuberculosis and non-tuberculous mycobacteria patients. Clin Exp Immunol. 2013; 171: 210-9.

64. Winau F, Weber S, Sad S, de Diego J, Hoops SL, Breiden B, et al. Apoptotic vesicles crossprime CD8 $\mathrm{T}$ cells and protect against tuberculosis. Immunity. 2006; 24: 105-17.

65. Rodrigues MF, Barsante MM, Alves CC, Souza MA, Ferreira AP, Amarante-Mendes GP, et al. Apoptosis of macrophages during pulmonary Mycobacterium bovis infection: correlation with intracellular bacillary load and cytokine levels. Immunology. 2009; 128: e691-9.

66. Thibodeau J, Bourgeois-Daigneault MC, Huppe G, Tremblay J, Aumont A, Houde $\mathrm{M}$, et al. Interleukin-10-induced MARCH1 mediates intracellular sequestration of MHC class II in monocytes. Eur J Immunol. 2008; 38: 1225-30.

67. Bobadilla K, Sada E, Jaime ME, Gonzalez Y, Ramachandra L, Rojas RE, et al. Human phagosome processing of Mycobacterium tuberculosis antigens is modulated by interferon-gamma and interleukin-10. Immunology. 2013; 138: $34-46$.

68. Giacomini E, Iona E, Ferroni L, Miettinen M, Fattorini L, Orefici G, et al. Infection of human macrophages and dendritic cells with Mycobacterium tuberculosis induces a differential cytokine gene expression that modulates $\mathrm{T}$ cell response. J Immunol. 2001; 166: 7033-41.

69. O'Leary S, O'Sullivan MP, Keane J. IL-10 blocks phagosome maturation in mycobacterium tuberculosis-infected human macrophages. Am J Respir Cell Mol Biol. 2011; 45: 172-80.

70. Meller S, Di Domizio J, Voo KS, Friedrich HC, Chamilos G, Ganguly D, et al. T17 cells promote microbial killing and innate immune sensing of DNA via interleukin 26. Nat Immunol. 2015.

71. Che KF, Tengvall S, Levanen B, Silverpil E, Smith ME, Awad M, et al. Interleukin-26 in antibacterial host defense of human lungs. Effects on neutrophil mobilization. Am J Respir Crit Care Med. 2014; 190: 1022-31.

72. Yeremeev V, Linge I, Kondratieva T, Apt A. Neutrophils exacerbate tuberculosis infection in genetically susceptible mice. Tuberculosis (Edinb). 2015; 95: 447-51.

73. Cilfone NA, Ford CB, Marino S, Mattila JT, Gideon HP, Flynn JL, et al Computational modeling predicts IL-10 control of lesion sterilization by balancing early host immunity-mediated antimicrobial responses with caseation during mycobacterium tuberculosis infection. J Immunol. 2015; 194: 664-77.

74. Qiu L, Huang D, Chen CY, Wang R, Shen L, Shen Y, et al. Severe tuberculosis induces unbalanced up-regulation of gene networks and overexpression of IL-22, MIP-1alpha, CCL27, IP-10, CCR4, CCR5, CXCR3，PD1，PDL2, IL-3, IFN-beta, TIM1, and TLR2 but low antigen-specific cellular responses. J Infect Dis. 2008; 198: 1514-9.

75. Bhuju S, Aranday-Cortes E, Villarreal-Ramos B, Xing Z, Singh M, Vordermeier HM. Global gene transcriptome analysis in vaccinated cattle revealed a dominant role of IL-22 for protection against bovine tuberculosis. PLoS Pathog. 2012; 8: e1003077.

76. Behrends J, Renauld JC, Ehlers S, Holscher C. IL-22 is mainly produced by IFNgamma-secreting cells but is dispensable for host protection against Mycobacterium tuberculosis infection. PLoS One. 2013; 8: e57379.

77. Khader SA, Guglani L, Rangel-Moreno J, Gopal R, Junecko BA, Fountain JJ, et al. IL-23 is required for long-term control of Mycobacterium tuberculosis and B cell follicle formation in the infected lung. J Immunol. 2011; 187: 5402-7.

78. Maglione PJ, Xu J, Chan J. B cells moderate inflammatory progression and enhance bacterial containment upon pulmonary challenge with Mycobacterium tuberculosis. J Immunol. 2007; 178: 7222-34.

79. Zhang G, Chen X, Chan L, Zhang M, Zhu B, Wang L, et al. An SNP selection strategy identified IL-22 associating with susceptibility to tuberculosis in Chinese. Sci Rep. 2011; 1: 20.

80. Scriba TJ, Kalsdorf B, Abrahams DA, Isaacs F, Hofmeister J, Black G, et al. Distinct, specific IL-17- and IL-22-producing CD4+ T cell subsets contribute to 
the human anti-mycobacterial immune response. J Immunol. 2008; 180: 1962-70.

81. Li L, Qiao D, Fu X, Lao S, Zhang X, Wu C. Identification of Mycobacterium tuberculosis-specific Th1, Th17 and Th22 cells using the expression of CD40L in tuberculous pleurisy. PloS one. 2011; 6: e20165.

82. Tadokera R, Wilkinson KA, Meintjes GA, Skolimowska KH, Matthews K, Seldon $\mathrm{R}$, et al. Role of the interleukin 10 family of cytokines in patients with immune reconstitution inflammatory syndrome associated with HIV infection and tuberculosis. J Infect Dis. 2013; 207: 1148-56.

83. van Laarhoven A, Mandemakers JJ, Kleinnijenhuis J, Enaimi M, Lachmandas E, Joosten LA, et al. Low induction of proinflammatory cytokines parallels evolutionary success of modern strains within the Mycobacterium tuberculosis Beijing genotype. Infect Immun. 2013; 81: 3750-6.

84. Zeng G, Chen CY, Huang D, Yao S, Wang RC, Chen ZW. Membrane-bound IL-22 after de novo production in tuberculosis and anti-Mycobacterium tuberculosis effector function of IL-22+ CD4+ T cells. J Immunol. 2011; 187: 190-9.

85. Dhiman R, Venkatasubramanian S, Paidipally P, Barnes PF, Tvinnereim A, Vankayalapati R. Interleukin 22 inhibits intracellular growth of Mycobacterium tuberculosis by enhancing calgranulin A expression. J Infect Dis. 2014; 209: 578-87.

86. Whitaker EL, Filippov VA, Duerksen-Hughes PJ. C. Cytokine Growth Factor Rev. 2012; 23: 323-31.

87. Wu B, Huang C, Garcia L, Ponce de Leon A, Osornio JS, Bobadilla-del-Valle M, et al. Unique gene expression profiles in infants vaccinated with different strains of Mycobacterium bovis bacille Calmette-Guerin. Infect Immun. 2007; 75: 3658-64.

88. Wu B, Huang C, Kato-Maeda M, Hopewell PC, Daley CL, Krensky AM, et al. Messenger RNA expression of IL-8, FOXP3, and IL-12beta differentiates latent tuberculosis infection from disease. J Immunol. 2007; 178: 3688-94. 